\title{
Raman Spectra from One Carbon Nanotube
}

\section{S. DRESSELHAUS ${ }^{a, b}$, A. JORIO ${ }^{b}$, A. G. SOUZA FILHO ${ }^{b, d}$, G. DRESSELHAUS ${ }^{c}$, R. SAITO ${ }^{e}$, and M. A. PIMENTA ${ }^{f}$}

${ }^{a}$ Dept. of Electrical Engineering and Computer Science, ${ }^{b}$ Dept. of Physics, ${ }^{c}$ Francis Bitter Magnet Laboratory, Massachusetts Institute of Technology, Cambridge, MA 02139-4307, USA; ${ }^{d}$ Dept. de Física, Univ. Federal do Ceará, Fortaleza - CE, 60455-760 Brazil; e Department of Electronic Engineering, University of Electro-Communications, Chofu, 182-8585 Tokyo, Japan; ${ }^{f}$ Dept. de Física, Univ. Federal de Minas Gerais, Belo Horizonte - MG, 30123-970 Brazil.

The use of Raman spectroscopy as a characterization tool for individual single wall carbon nanotubes is briefly reviewed. New physical phenomena occurring at the single nanotube level are discussed, with special emphasis given to the use of resonance Raman scattering for the structural determination of $(n, m)$ for individual nanotubes, based on diameter and chirality dependent phenomena associated with the radial breathing mode, the G-band and the $\mathrm{G}^{\prime}$-band features. Examples are given to show how single nanotube spectroscopy provides insight into the use of Raman spectroscopy for the characterization of nanotube bundles and for the study of new physical phenomena occurring at the single nanotube level.

Keywords: carbon nanotubes, Raman spectroscopy, Raman characterization, radial breathing mode, D-band, double resonance, dispersive phonon modes.

\section{INTRODUCTION}

The ability to carry out Raman scattering studies at the single nanotube level and to use such spectra to identify the $(n, m)$ structural indices for an individual nanotube opens up many new possibilities for the discovery of new physical phenomena never seen before in any system, for understanding how the Raman spectra from single wall carbon nanotube (SWNT) bundles are related to the constituent SWNTs in the bundle, and for using the Raman characterization technique to carry out studies of other physical properties of SWNTs of known $(n, m)$ values at the single nanotube level. The use of Raman spectroscopy for determining nanotube diameters and for distinguishing between metallic and semiconducting SWNTs is well known from measurements on SWNT bundles [1]. In the present review, we focus on the wealth of additional information provided by spectroscopy at the single nanotube level.

The $(n, m)$ integers are conventionally used to specify the number of unit vectors $\vec{a}_{1}$ and $\vec{a}_{2}$ in the graphene honeycomb structure that constitute the chiral vector (or roll-up vector) $\overrightarrow{\mathcal{C}_{h}}=n \vec{a}_{1}+m \vec{a}_{2}$ corresponding to the nanotube circumference [2]. Once $(n, m)$ is known, the nanotube diameter $d_{t}$ and chirality, or the orientation of the carbon hexagons with respect to the nanotube axis, can be specified [2]. Raman spectra of the radial breathing mode, whereby each carbon atom in the nanotube vibrates in phase in the radial direction, give a direct measure of $d_{t}$, because the radial breathing mode frequency $\omega_{\mathrm{RBM}}$, is given by the relation $\omega_{\mathrm{RBM}}=\alpha / d_{t}$, where $\alpha$ is found to be $248 \mathrm{~cm}^{-1} \mathrm{~nm}$, based 
on measurements of many SWNTs at the single nanotube level [3].

The electronic properties of SWNTs are remarkable insofar as they can be either metallic (when $m-n=3 q$ and $q$ is an integer) or semiconducting (when $n-m=3 q \pm 1$ ). Raman spectroscopy can be used to distinguish between metallic and semiconducting tubes, because of the very different spectral lineshapes observed for metallic and semiconducting SWNTs with diameters less than $2 \mathrm{~nm}[1]$.

\section{Single Nanotube Raman Spectroscopy}

The large density of electronic states for one-dimensional (1D) systems at van Hove singularities [see Fig. 1(b)] and the strong electron-phonon coupling in carbon nanotubes under resonance conditions allows observation of the Raman spectra from one individual single wall carbon nanotube [see Fig. 1(d)], when the incident or scattered photon is in resonance with an interband transition $E_{i i}$ between the $i$-th 1D van Hove singularities in the electron density of states of the valence and conduction bands. The enhancement of the Raman signal coming from the resonance between the excitation photon and these singularities in the joint density of states (JDOS) can be very large, so that in some cases the Raman intensity from one nanotube can be as large as that for the silicon substrate on which the nanotubes lie [see Fig. 1(e)], even though there is a $10^{8}$ ratio of $\mathrm{Si} / \mathrm{C}$ atoms within the laser excitation beam of $1 \mu \mathrm{m}$ diameter [see inset to Fig. 1(d) where the radial breathing mode features for 3 different SWNTs are shown].

Such large enhancement factors arise from the highly 1D nature of the electronic density of states of carbon nanotubes with diameters less that $\sim 2 \mathrm{~nm}$. Figure $1(\mathrm{~d})$ shows that each feature in the Raman spectra of SWNT bundles can now be observed at the single nanotube level. These features include the non-dispersive radial breathing mode (RBM) which is not present in other $s p^{2}$ carbons, and the non-dispersive G-band feature, which is also present in $s p^{2}$ carbons and has many properties for nanotubes that are both similar to and distinct from the G-band features in other $s p^{2}$ carbons. The RBM and G-band processes are both first-order Raman processes, for which the resonance can be with either the incident or the scattered photon. Also seen in the Raman spectra in Fig. 1(d) are the highly dispersive disorder-induced D-band and its second harmonic $\mathrm{G}^{\prime}$-band, for which the mode frequencies show a strong dependence on the laser excitation energy (for the $G^{\prime}$-band measured in SWNT bundles, $\partial \omega_{G^{\prime}} / \partial E_{\text {laser }}=106 \mathrm{~cm}^{-1} / \mathrm{eV}$ ). Investigations of these spectral features at the single nanotube level reveal many interesting details about the dependence of each feature in Fig. 1(d) on nanotube diameter, chirality, metallic vs. semiconducting behavior. In addition, such phonon spectra provide an astonishingly sensitive probe of the unique electronic structure of single wall carbon nanotubes. Furthermore, study of the Raman spectra at the single nanotube level allows investigation of new physical phenomena, particularly phenomena about the resonance Raman effect that have never been observed before in any physical system.

Raman spectroscopy is not normally a tool in solid state physics for the structural characterization of crystalline solids, but for the case of 1D carbon nanotubes, the observation of the Raman spectra from an individual nanotube can be used to provide a definitive identification of the nanotube structure through determination of its $(n, m)$ indices. This is another remarkable and unique property of carbon nanotubes. The physical principle that is employed in the $(n, m)$ determination makes use of the fact that because of the trigonal warping effect of the equi-energy contours around the $\mathrm{K}$ and $\mathrm{K}^{\prime}$ points in the $2 \mathrm{D}$ Brillouin zone [Fig. 1(a)], each $(n, m)$ nanotube has a unique set of singularities [Fig. 1(b)], at energies $E_{i i}$ in the JDOS [Fig. 1(c)], where each $E_{i i}$ value is plotted for the indicated 
(a)

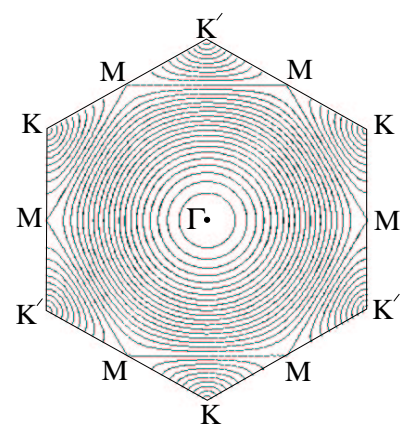

(d)

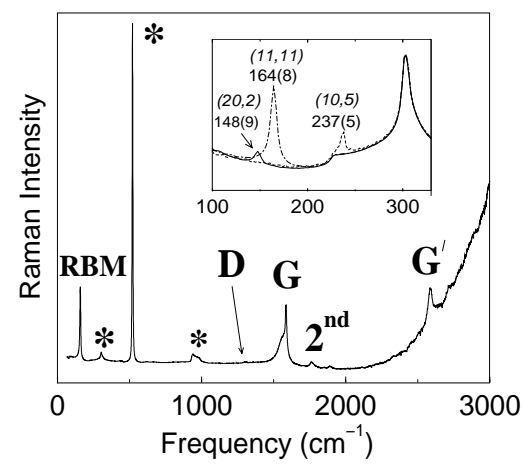

(b)

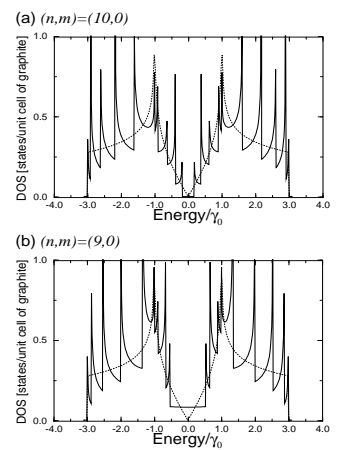

(e)

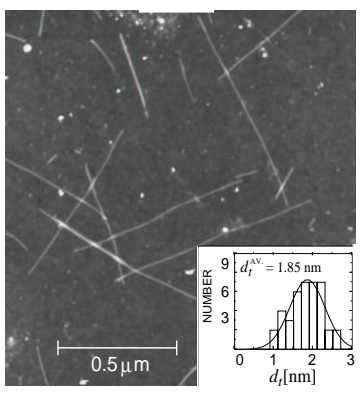

(c)

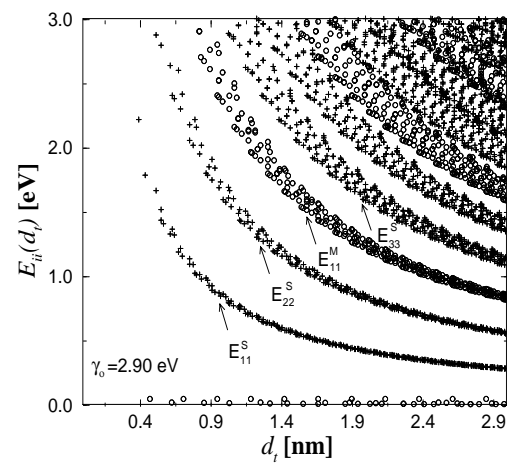

(f)
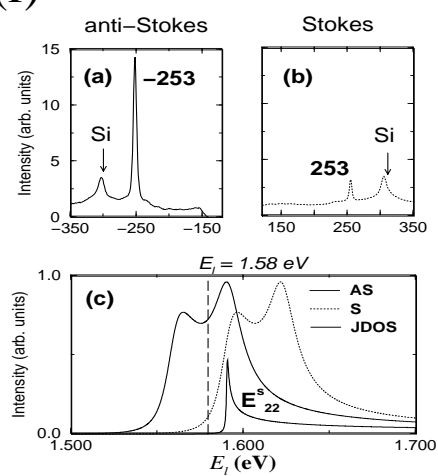

Figure 1: (a)Plot of the 2D equi-energy contours of graphite, showing trigonal warping effects, as we move from the $K$ point in the $K-\Gamma$ or $K-M$ directions. The equi-energy contours are circles near the $K$ point and near the center of the 2D Brillouin zone. But near the zone boundary, the contours are straight lines which connect the nearest $M$ points $[4,5]$. (b)The density of electronic states for a semiconducting $(10,0)$ and a metallic $(9,0)$ SWNT showing the van Hove singularities. The dotted curves show the corresponding density of states for 2D graphite [2]. (c) Calculated [6] energy separations $E_{i i}\left(d_{t}\right)$ between van Hove singularities $i$ in the 1D electronic density of states of the conduction and valence bands for all $(n, m)$ SWNTs vs. nanotube diameter for $0.4<d_{t}<3.0 \mathrm{~nm}$, using a value for the carbon-carbon energy overlap integral of $\gamma_{0}=2.9 \mathrm{eV}$ and a nearest neighbor carboncarbon distance $a_{\mathrm{C}-\mathrm{C}}=1.42 \AA[4,7]$. Semiconducting (S) and metallic (M) nanotubes are indicated by crosses and open circles, respectively. The index $i$ in the interband transitions $E_{i i}$ denotes the transition between the van Hove singularities, with $i=1$ being closest to the Fermi level. (d) Raman spectrum from one nanotube taken over a broad frequency range using $E_{\text {laser }}=785 \mathrm{~nm}=1.58 \mathrm{eV}$ excitation, and showing the radial breathing mode (RBM), the D-band, the G-band, second order features and the $\mathrm{G}^{\prime}$-band. The features marked with '*) at $303 \mathrm{~cm}^{-1}, 521 \mathrm{~cm}^{-1}$ and $963 \mathrm{~cm}^{-1}$ [8] are from the $\mathrm{Si} / \mathrm{SiO}_{2}$ substrate and are used for calibration. The inset shows the RBM feature for 3 different SWNTs in comparison to the Si feature at $303 \mathrm{~cm}^{-1}$. (e)AFM image of the sample used for single nanotube spectroscopy showing isolated SWNTs grown from the vapor phase [9]. The small particles are iron catalyst particles. The inset shows the diameter distribution of this sample based on AFM observations of $40 \mathrm{SWNTs}$ on a $\mathrm{Si} / \mathrm{SiO}_{2}$ surface [3]. (f)AntiStokes and Stokes spectra for a $(12,1)$ SWNT identified from the corresponding calculated intensities shown below, based on energy difference between $E_{\text {laser }}$ and $E_{22}^{S}$ [10]. 
range of nanotube diameters $d_{t}$. Each point on a $E_{i i}^{S}$ band in Fig. 1(c) corresponds to a different semiconducting nanotube, and each metallic nanotube has a pair of points on each $E_{i i}^{M}$ band in Fig. 1(c) because of the trigonal warping effect [4], except for armchair $(n, n)$ SWNTs for which the trigonal warping effect goes to zero. Resonance Raman scattering allows determination of these energies $E_{i i}$ and the corresponding wave vectors $k_{i i}$ where these van Hove singularities occur, because the energy differences $E_{i i}$ between the $i$-th van Hove singularities in the valence and conduction bands control the resonant condition of the SWNT with the laser excitation energy $E_{\text {laser }}$. The determination of $E_{i i}$ by the Raman experiment, in turn, provides a determination of the $(n, m)$ indices of the nanotube in resonance with $E_{\text {laser }}$. The $E_{i i}$ value is most directly determined (to an accuracy of $\sim 10 \mathrm{meV}$ ) from measurement of the Stokes and anti-Stokes radial breathing mode intensities as shown in Fig. 1(f) [10]. Here we see the anti-Stokes and Stokes spectra for a $(12,1)$ SWNT, which is identified by reproducing the measured intensity ratio by fitting the energy of the $E_{22}^{S}$ singularity in the JDOS. When the Stokes/anti-Stokes measurements are made with a tunable laser, then $E_{i i}$ can be determined to a resolution of $3 \mathrm{meV}$ [11]. The tunable laser study also allows measurement of the profile of the joint density of states near one of its characteristic 1D singularities, showing that the width of the $1 \mathrm{D}$ singularities in the density of states is less than $1 \mathrm{meV}$, thereby explaining how it is possible to get a sharp Raman signal from a nanostructure as small as one SWNT. When characterizing many SWNTs for their $(n, m)$ values, the measurements are made in the simplest way, by measuring the radial breathing mode frequency $\left(\omega_{\mathrm{RBM}}\right)$ and intensity $\left(I_{\mathrm{RBM}}\right)$ using one laser line. Such a measurement is sufficient to determine $(n, m)$ in about $90 \%$ of the cases. For a more precise $(n, m)$ determination, either the Stokes/anti-Stokes intensity ratio can be used [10], or measurements can be made of the properties of other Raman features that are sensitive to diameter and chirality, some of which are summarized below. Because of the high sensitivity of the electronic, transport, vibrational and other nanotube properties to the structural $(n, m)$ indices, this non-destructive, readily available resonance Raman characterization technique is expected to have a significant impact on current basic research on carbon nanotubes generally, because now it will be possible to study many properties at the single nanotube level as a function of diameter and chirality by combining resonance Raman spectroscopy with other measurement techniques [12]. A few examples of the richness of this newly emerging field of single nanotube spectroscopy are presented in this brief review.

\section{Connecting Single Nanotube and Bundle Spectra}

Figure 2 gives three examples of what single nanotube spectroscopy tells us about the spectra of SWNT bundles. Measurements of the radial breathing mode at the single nanotube level yields a relatively narrow Lorentzian line [see Fig. 2(a)]. A determination of the coefficient $\alpha=248 \mathrm{~cm}^{-1} \mathrm{~nm}$ in the relation for the nanotube diameter, $d_{t}=248 / \omega_{\mathrm{RBM}}$ is found by RBM measurements on many nanotubes in conjunction with a determination of their $(n, m)$ indices [3]. The spectrum for the SWNT bundle in contrast shows a superposition of Raman signals from all tubes resonant with $E_{\text {laser }}=1.58 \mathrm{eV}$. Spectra at

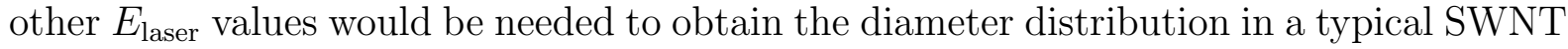
bundle sample, since only a fraction of the SWNTs in the bundle are in resonance with a given $E_{\text {laser }}$ line.

As another example of what can be learned by single nanotube spectroscopy, we consider the G-band feature. The difference in lineshape of the G-band feature between metallic and semiconducting SWNTs can be easily seen at the individual nanotube level 

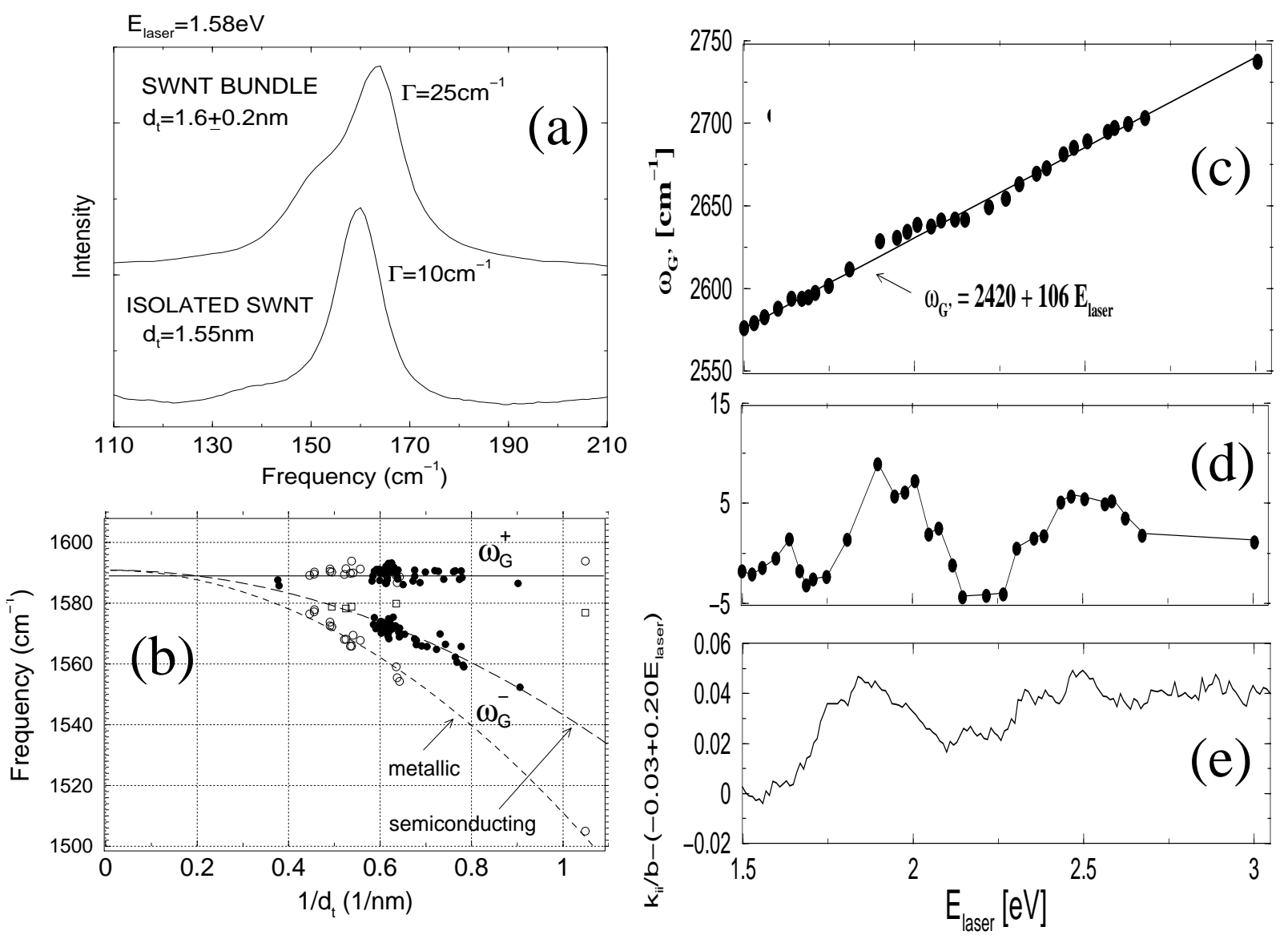

Figure 2: (a) RBM Raman spectra for one isolated nanotube and for SWNT bundles. (b)Splitting of the G-band frequency showing the diameter dependence of the circumferential and axial mode frequencies $\omega_{G}^{+}$and $\omega_{G}^{-}$for metallic and semiconducting SWNTs [13]. (c) $\mathrm{G}^{\prime}$-band data for $\omega_{G^{\prime}}$ for a SWNT bundle sample taken from Ref. [14]. (d) Same $\omega_{G^{\prime}}$ vs. $E_{\text {laser }}$ data as in (c), after subtracting the linear dispersion $2420+106 E_{\text {laser }}$. (e) Calculated first moment $\bar{k}_{i i}$ for all possible resonant tubes as a function of $E_{\text {laser }}$ after subtracting the linear dispersion $-0.03+0.20 E_{\text {laser }}$, and normalizing $\bar{k}_{i i}$ to $\mathrm{b}$, the magnitude of the basis vector in the $2 \mathrm{D}$ reciprocal graphene lattice [15]. 
[16]. It is only at the single nanotube level that the dependence of the G-band frequency on nanotube diameter $d_{t}$ can be measured quantitatively [see Fig. 2(b)], where it is seen that $\omega_{G}^{+}$for vibrations along the nanotube axis is independent of $d_{t}$ and is the same for semiconducting and metallic SWNTs. On the other hand, the frequency for the vibrations in the circumferential direction $\omega_{G}^{-}$show a $\mathcal{C} / d_{t}^{2}$ dependence, where the value of $\mathcal{C}$ is nearly twice as large $\mathcal{C}_{M}=79.5 \mathrm{~cm}^{-1} \mathrm{~nm}^{2}$ for metallic SWNTs as for semiconducting SWNTs $\left(\mathcal{C}_{S}=47.7 \mathrm{~cm}^{-1} \mathrm{~nm}^{2}\right)[13]$. Furthermore, no chirality dependence is observed for $\omega_{G}^{ \pm}$, so that their measurement does not provide a sensitive determination of $(n, m)$. On the other hand, one use of the $d_{t}$ dependence of $\omega_{G}^{-}$is for the analysis of Raman spectra from SWNT bundles in the estimation of the mean diameters for semiconducting and metallic nanotubes contained in the bundle. For individual SWNTs in resonance with the incident $E_{\text {laser }}$, measurements of $\omega_{G}^{ \pm}$can be used as an internal check on the diameter determination made by analysis of the radial breathing mode [17]. Another application of the $d_{t}$ dependence of the $\omega_{G}^{-}$measurement is for estimating $d_{t}$ for SWNTs that are in resonance with the scattered photon, so that the radial breathing mode would not in this case be in resonance with $E_{\text {laser }}$, and there is therefore no RBM spectrum available for an $(n, m)$ identification.

Polarization effects are very important for Raman intensity measurements because of the strong polarization effect of SWNTs in aligning the optical $E$ field with the SWNT axis. This effect can also be used to align SWNTs along the polarization direction of $E_{\text {laser }}[18]$, and this procedure is very important for comparing the intensities of specific features in single nanotube spectroscopy experiments on different SWNTs. Polarization experiments made on two SWNTs that lie close (less than an optical wavelength away) to each other on the substrate, but have different spatial directions for their axes, show anomalous polarization effects. For such tubes, the complete suppression of the Raman intensity for the polarization perpendicular to each of the tubes is not observed because of the complicated form of the antenna effect that is produced in this case. Such observations at the single nanotube level clarify the physical phenomena occurring in polarization experiments done on SWNT bundles where the effect of the local optical field due to the antenna effect of each nanotube must be considered in interpreting the polarization spectrum.

The third example concerns the very large dispersion of the $G^{\prime}$-band frequency $\omega_{G^{\prime}}$ as a function of $E_{\text {laser }}$ for a SWNT bundle [14]. This example involves the highly dispersive features of the Raman spectra from isolated SWNTs associated with the D-band and the $\mathrm{G}^{\prime}$-band. The origin of the disorder-induced Raman peak (D-band) which is observed around $1350 \mathrm{~cm}^{-1}$ for laser excitation energy $E_{\text {laser }}=2.41 \mathrm{eV}[1,16,19]$ in both graphite and single wall carbon nanotubes $[20,21]$ has been explained by a double resonance Raman process [21]. This double resonance process in graphite is a second-order scattering process, and a Raman intensity enhancement occurs due to resonance with both an intermediate state and a second resonance occurring with either the initial or final states, thus yielding a double resonance process for which the phonon wave vector at resonance is twice the electron wave vector $(q \simeq 2 k)[20,21]$. For SWNTs, the van Hove singularities sensitively determine the $k_{i i}$ wave vectors for the resonance condition [20].

After subtracting the strong linear dependence of $\omega_{G^{\prime}}$ on $E_{\text {laser }}$, that is also observed with approximately the same large magnitude as for other $s p^{2}$ carbons [see Fig. 2(c)], an oscillatory component unique to the $\omega_{G^{\prime}}\left(E_{\text {laser }}\right)$ dispersion for SWNTs remains, as shown in Fig. 2(d). The physical basis of these oscillations stems from the special role of the singularities of the 1D JDOS in the electronic structure of SWNTs in the double resonance mechanism for the $G^{\prime}$ band as it applies to SWNTs [22]. The general dispersive 
nature of $\omega_{G^{\prime}}$ as a function of $E_{\text {laser }}$ arises from the double resonance process in $s p^{2}$ carbons [21]. In the basic second-order scattering process for the $G^{\prime}$-band in the $s p^{2}$ carbons, an electron with momentum $k$ near the K-point in the 2D Brillouin zone (a) is excited by the incident photon. (b) This electron is then scattered to an intermediate state $k+q$ state and (c) finally is back-scattered to the $k$ state to recombine with a hole. If two of three intermediate states correspond to real electronic states in the energy dispersion $E(k)$, the Raman intensity is enhanced by two factors in the denominator of the intensity formula, thereby constituting the double resonance Raman process. For the $G^{\prime}$-band process, two phonons of wave vector $q$ and $-q$ are emitted in the second-order Stokes scattering process $[20]$.

In addition to the double resonance process for $s p^{2}$ carbons, the singularities in the $1 \mathrm{D}$ electronic JDOS govern the specific nanotubes in the SWNT bundle that are in resonance with $E_{\text {laser }}$, namely SWNTs that have $E_{i i}$ singularities close in energy to $E_{\text {laser }}$. The $k_{i i}$ wave vector corresponding to each $E_{i i}$ value will strongly couple to a phonon with wave vector $q \simeq 2 k_{i i}$. For a SWNT bundle, each $E_{i i}$ band for metallic or semiconducting SWNTs [see Fig. 1(c)] gives rise to an oscillatory behavior in the $\omega_{G^{\prime}}$ vs. $E_{\text {laser }}$ dispersion curve, with the oscillation centered at $1.9 \mathrm{eV}$ associated with $E_{11}^{M}$ interband transitions and the oscillation centered at $2.5 \mathrm{eV}$ associated with the $E_{33}^{S}$ and $E_{44}^{S}$ interband transitions which lie too close together in energy to be separated in Fig. 2(d). For each SWNT in the bundle, resonance is possible with either the incident photon, the scattered photon or the intermediate state $k_{i i}+q$ resulting from the phonon scattering event in the double scattering process. By considering all possible SWNTs in the SWNT bundle that can contribute to each of the $E_{11}^{M}, E_{33}^{S}$ and $E_{44}^{S}$-bands, the calculated curve shown in Fig. 2(e), is obtained. The behavior in Figs. 2(d) and 2(e) arises from the detailed dependence of the $k_{i i}$ values on nanotube diameter $\left(\omega_{G^{\prime}}\right.$ decreases as $d_{t}$ decreases $)$ and on chiral angle, and both of these effects have been calculated [15, 23].

From these studies of the dependence of the $\mathrm{G}^{\prime}$-band feature on nanotube diameter and chirality at the single nanotube level, together with gaining an understanding of the role of the van Hove singularities in the double resonance process, we learn that the $\mathrm{G}^{\prime}$-band for SWNT bundles is diameter selective in accordance with strong resonances with transitions between van Hove singularities. We further learn that not all SWNTs contribute resonantly to the $\mathrm{G}^{\prime}$-band at every $E_{\text {laser }}$ value, as occurs for graphite and $s p^{2}$ carbons. Studies at the single nanotube level also provide an explanation for the oscillatory behavior superimposed on the strong linear behavior of $\omega_{G^{\prime}}$ vs. $E_{\text {laser }}$ observed for SWNT bundles [15].

\section{Unique Effects in Isolated SWNTs}

In this section we give four examples of new phenomena in resonant Raman spectroscopy that have been observed for the first time in carbon nanotubes. All of these examples depend strongly upon selecting SWNTs with special $(n, m)$ values which exhibit unique phenomena. The good agreement between predictions and experimental observations for each of these examples thus provides independent confirmation of the $(n, m)$ assignment, made primarily from analysis of the RBM feature.

The first example involves the dependence of the Stokes and anti-Stokes intensities for the radial breathing mode on a tunable laser excitation energy, as shown in Fig. 3(a). Here the relative intensities of the anti-Stokes to Stokes features are not governed by the Boltzmann factor alone, but also by the difference in energy between the van Hove singularity and the laser line. By using a tunable laser source, the radial breathing mode 
(a)

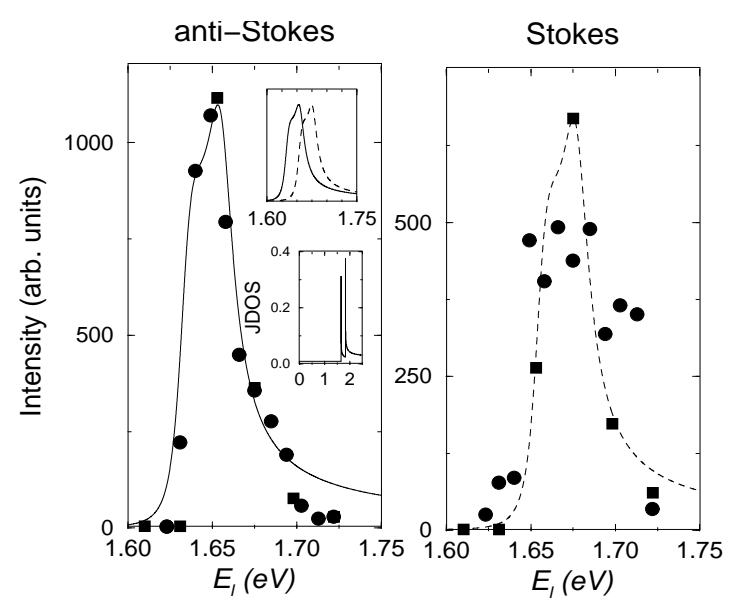

(c)

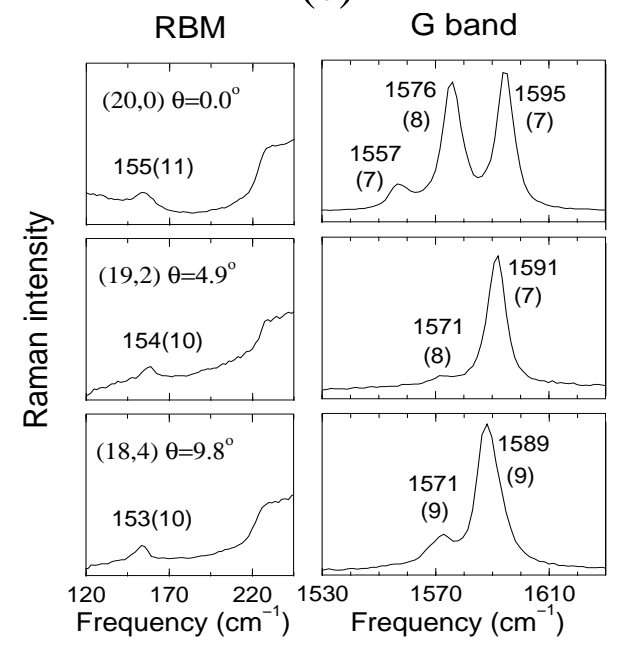

(b)
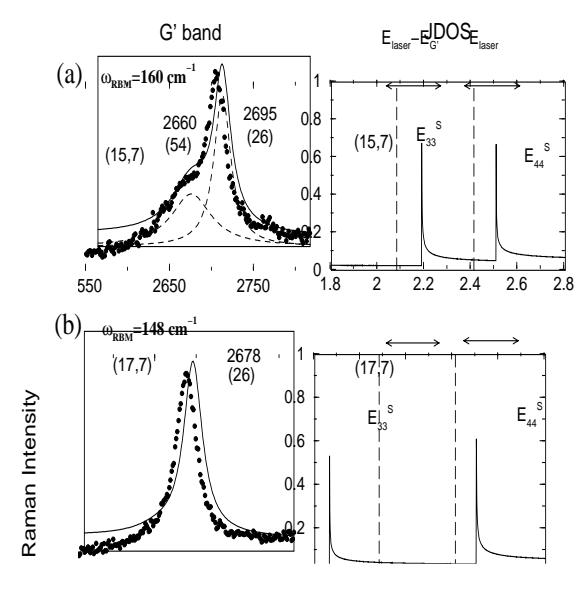

(d)

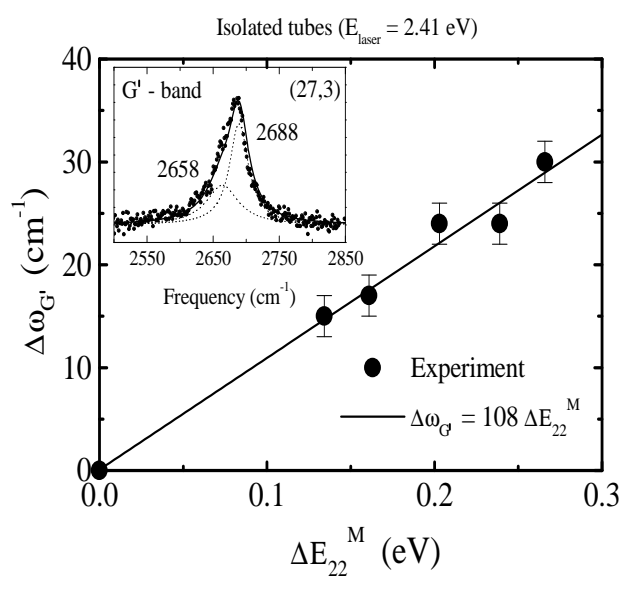

Figure 3: (a)Raman intensity vs. laser excitation energy $E_{\text {laser }}$ for the radial breathing mode $\left(\omega_{\mathrm{RBM}}=173.6 \mathrm{~cm}^{-1}\right.$ peak $)$ in the Stokes and anti-Stokes Raman processes of an isolated $(18,0) \mathrm{SWNT}$, where the anti-Stokes intensity is normalized by $\exp \left(\hbar \omega_{\mathrm{RBM}} / k_{B} T\right)$ to facilitate comparisons with the Stokes profile. The curves indicate the predicted resonant Raman windows. The upper inset compares the theoretically predicted Stokes and anti-Stokes resonant windows. The lower inset plots the JDOS profile for one isolated $(18,0)$ SWNT [11]. (b) $G^{\prime}$-band profile (left panel) and joint density of electronic states (right panel) for the $(15,7)$ and $(17,7)$ SWNTs. The incident photon for $E_{\text {laser }}=2.41 \mathrm{eV}$ is resonant with $E_{44}^{S}$ for both the $(15,7)$ and $(17,7)$ SWNTs. The scattered photon at $E_{\text {laser }}-E_{G^{\prime}} \approx 2.08 \mathrm{eV}$ is resonant with $E_{33}^{S}$ for the $(15,7)$ SWNT, but not for the $(17,7)$ tube. The vertical dashed lines at $2.41 \mathrm{eV}$ and $2.08 \mathrm{eV}$ are for the incident and scattered photons, respectively. The horizontal double arrows denote the resonant windows for the incident and scattered photons [15, 22]. (c) RBM and the G-band Raman spectra for three isolated semiconducting SWNTs with similar diameters $\left(d_{t} \sim 1.61 \mathrm{~nm}\right)$ that are resonant with the laser $\left(E_{\text {laser }}=2.41 \mathrm{eV}\right)$ at three different spots on the sample. The shoulder observed to the right side of the RBM spectral feature comes from the Si substrate [13]. (d) Linear fit for several SWNTs of the $\Delta \omega_{G^{\prime}}$ phonon splitting (experiment) vs. the electronic $\Delta E_{22}^{M}$ splitting (calculated) due to the chirality-dependent trigonal warping effect in metallic SWNTs. The inset is the $\mathrm{G}^{\prime}$-band profile for a $(27,3)$ SWNT showing the two-peak structure associated with the trigonal warping effect $[22,23]$. 
of the $(18,0)$ SWNT was measured as it moved through its resonant window, allowing measurement of the profile of the van Hove singularity in the joint density of states for this SWNT. The upper inset to Fig. 3(a) shows the shift in the windows for the Stokes and anti-Stokes processes and the lower inset shows the van Hove singularities in the joint density of states associated with the $E_{11}^{M}$ interband transitions. Measurement of the full profile of the radial breathing mode provides a determination of $E_{11}^{M}$ for this nanotube to an accuracy of $\sim 3 \mathrm{meV}$.

The second example shown in Fig. 3(c), illustrates how the G-band spectrum can in some special cases be used more directly to confirm the $(n, m)$ identification for some special range of SWNT diameters where $E_{\text {laser }}$ for the incident photon happens to be in resonance with one $E_{i i}$ van Hove singularity in the JDOS and $E_{\text {laser }}-E_{G}$ for the scattered photon is in resonance with another $E_{i i}$ for the same SWNT [13]. An example of such a situation occurs for $E_{\text {laser }}=2.41 \mathrm{eV}$, where a few special tubes with $d_{t} \simeq 1.61 \mathrm{~nm}$ show resonance of the incident photon with $E_{44}^{S}$ and of the scattered photon with $E_{33}^{S}$. It should be pointed out that these two processes are independent and involve different electron-hole pairs for each process, occurring at different $E_{i i}$ singularities. The special $(n, m)$ nanotubes exhibiting this effect for a given laser excitation energy $E_{\text {laser }}$ can be predicted theoretically. The experimental observation of this effect is in terms of unusual relative magnitudes of the main lower and upper components $\left(\omega_{G}^{-}\right.$and $\left.\omega_{G}^{+}\right)$of the G-band feature, as well as an exceptionally strong intensity for the $E_{2}$ symmetry component of the G-band, which is generally very difficult to observe, but can be seen in Fig. 3(c) at $1557 \mathrm{~cm}^{-1}[13]$.

The large dispersion of the $G^{\prime}$ band allows observation of new physical phenomena, not seen in other physical systems. For example, for the case of semiconducting nanotubes, it is possible at the single nanotube level for the incident photon to be resonant with the $E_{44}^{S}$ singularity for that SWNT and the scattered photon to be resonant with $E_{33}^{S}$. This effect can occur for a very special subset of SWNTs that can be predicted theoretically. The experimental manifestation of this phenomenon in semiconducting tubes appears as a two peak structure in the $\mathrm{G}^{\prime}$-band spectra, as shown in the top of Fig. 3(b). Also shown in the figure are the energies of the van Hove singularities in the joint density of states for $E_{44}^{S}$ and $E_{33}^{S}$, as well as $E_{\text {laser }}$ and $E_{\text {laser }}-E_{G^{\prime}}$. This special subset of SWNTs that exhibit this effect is strongly dependent on their $(n, m)$ assignments [12]. The excellent agreement between experiment and theory as to which semiconducting SWNTs show this two peak structure [such as the $(15,7)$ SWNT shown in the top of Fig. 3(b)] and which SWNTs do not, [the $(17,7)$ SWNT shown in the bottom of Fig. 3(b)] gives strong confirmation for the method used to obtain the $(n, m)$ assignments from analysis of the radial breathing mode feature. The presence or absence of the two peak structure can then be used as a method for distinguishing between two $(n, m)$ identifications when the nanotube diameters for the two choices of $(n, m)$ are very close to one another [15, 22].

For the Raman spectra for metallic SWNTs at the single nanotube level, the splitting of the singularities in the electronic JDOS due to the trigonal warping effect [see Fig. 1(a)] [4] can be measured quantitatively by measuring the corresponding splitting in $\omega_{G^{\prime}}$ denoted by $\Delta \omega_{G^{\prime}}$, and associated with each of the two components in the splitting of the electronic states $\Delta E_{i i}^{M}$ for metallic nanotubes, where $\Delta E_{i i}^{M}$ is zero for armchair SWNTs and is a maximum for zigzag SWNTs. Since the different electronic energies occur for each component of $\Delta E_{i i}^{M}$ in the trigonally split singularities in the JDOS [4], different $k_{i i^{-}}$ vectors will result, thereby giving rise to different $q$ values for the $G^{\prime}$ band phonons and finally different $\omega_{G^{\prime}}$ values will be obtained, thus resulting in the two peak structure $\Delta \omega_{G^{\prime}}$ shown in the inset to Fig. 3(d). By measuring these splittings $\Delta \omega_{G^{\prime}}$ in the $\omega_{G^{\prime}}$ profiles 
for SWNTs with different chiralities, it is possible to determine the dependence of the experimental $\Delta \omega_{G^{\prime}}$ on the calculated $\Delta E_{i i}^{M}$, as shown in Fig. 3(d) [23]. The linear relation observed experimentally for $\Delta \omega_{G^{\prime}}$ vs. $\Delta E_{i i}^{M}$ for various SWNTs gives an independent determination of $108 \pm 5 \mathrm{~cm}^{-1} / \mathrm{eV}$ for the dispersion of $\omega_{G^{\prime}}$ as a function of the excitation energy $E_{\text {laser }}$, even though the measurements on the various SWNTs are all made using one laser line $[22,23]$. This result is also important for understanding the relation between single nanotube spectroscopy and the Raman spectra obtained from SWNT bundles. The consistency of these two determinations of the dispersion of $\omega_{G^{\prime}}$ vs. $E_{\text {laser }}$ gives further support for the method used for the $(n, m)$ determination, since the $(n, m)$ identification of each SWNT measured in Fig. 3(d) sensitively determines its $\Delta E_{i i}^{M}$ value.

\section{Conclusion}

In conclusion, single nanotube spectroscopy opens up many new research directions, leading to the discovery of many new physical phenomena, resulting in a better understanding of what is actually observed in the Raman spectroscopy of SWNT bundles, and providing a basis for using Raman characterization to carry out quantitative studies of the dependence of other physical phenomena (e.g., transport, mechanical, thermal properties) on nanotube diameter and chirality at the single nanotube level.

The authors thank many colleagues for their contributions to this research. R.S. acknowledges a Grant-in-Aid (No. 13440091) from the Ministry of Education, Japan. A.J. and M.A.P./A.G.S.F. acknowledge support from the Brazilian agencies CNPq/CAPES. The MIT authors acknowledge support under NSF Grants DMR 01-16042, INT 98-15744, and INT 00-00408.

\section{REFERENCES}

[1] M. S. Dresselhaus and P. C. Eklund, Advances in Physics 49, 705-814 (2000).

[2] R. Saito, G. Dresselhaus, and M. S. Dresselhaus, Physical Properties of Carbon Nanotubes (Imperial College Press, London, 1998).

[3] A. Jorio, R. Saito, J. H. Hafner, C. M. Lieber, M. Hunter, T. McClure, G. Dresselhaus, and M. S. Dresselhaus, Phys. Rev. Lett. 86, 1118-1121 (2001).

[4] R. Saito, G. Dresselhaus, and M. S. Dresselhaus, Phys. Rev. B 61, 2981-2990 (2000).

[5] T. W. Odom, J. L. Huang, P. Kim, and C. M. Lieber, Nature (London) 391, 62-64 (1998).

[6] H. Kataura, Y. Kumazawa, Y. Maniwa, I. Umezu, S. Suzuki, Y. Ohtsuka, and Y. Achiba, Synthetic Metals 103, 2555-2558 (1999).

[7] G. Dresselhaus, M. A. Pimenta, R. Saito, J.-C. Charlier, S. D. M. Brown, P. Corio, A. Marucci, and M. S. Dresselhaus. In Science and Applications of Nanotubes, edited by D. Tománek and R. J. Enbody, pages 275-295, Kluwer Academic, New York, 2000. Proceedings of the International Workshop on the Science and Applications of Nanotubes, Michigan State University, East Lansing, MI, USA, July 24-27, 1999.

[8] P. A. Temple and C. E. Hathaway, Phys. Rev. B 7, 3685-3697 (1973). 
[9] J. H. Hafner, C. L. Cheung, T. H. Oosterkamp, and C. M. Lieber, J. Phys. Chem. B 105, 743 (2001).

[10] A. G. Souza Filho, A. Jorio, J. H. Hafner, C. M. Lieber, R. Saito, M. A. Pimenta, G. Dresselhaus, and M. S. Dresselhaus, Phys. Rev. B 63, 241404R (2001).

[11] A. Jorio, A. G. Souza Filho, G. Dresselhaus, M. S. Dresselhaus, R. Saito, J. H. Hafner, C. M. Lieber, F. M. Matinaga, M. S. S. Dantas, and M. A. Pimenta, Phys. Rev. B 63, 5416 (2001).

[12] A. Jorio, F. M. Matinaga, A. Righi, M. S. S. Dantas, M. A. Pimenta, A. G. Souza Filho, J. Mendes Filho, J. H. Hafner, C. M. Lieber, R. Saito, G. Dresselhaus, and M. S. Dresselhaus, (to be published).

[13] A. Jorio, A. G. Souza Filho, G. Dresselhaus, M. S. Dresselhaus, A. K. Swan, B. Goldberg, M. S. Ünlü, M. A. Pimenta, J. H. Hafner, C. M. Lieber, and R. Saito, (to be published).

[14] M. A. Pimenta, E. B. Hanlon, A. Marucci, P. Corio, S. D. M. Brown, S. A. Empedocles, M. G. Bawendi, G. Dresselhaus, and M. S. Dresselhaus, Brazilian J. Phys. 30, 423-427 (2000).

[15] A. G. Souza Filho, A. Jorio, G. Dresselhaus, M. S. Dresselhaus, Anna K. Swan, M. S. Ünlü, B. B. Goldberg, R. Saito, J. H. Hafner, C. M. Lieber, and M. A. Pimenta, (to be published).

[16] M. S. Dresselhaus, G. Dresselhaus, A. Jorio, A. G. Souza Filho, and R. Saito, Carbon (to be published) (2002).

[17] M. Milnera, J. Kurti, M. Hulman, and H. Kuzmany, Phys. Rev. Lett. 84, 1324-1327 (2000).

[18] A. Jorio, A. G. Souza Filho, V. W. Brar, A. K. Swann, M. S. Ünlü, B. B. Goldberg, A. Righi, J. H. Hafner, C. M. Lieber, R. Saito, G. Dresselhaus, and M. S. Dresselhaus, (to be published).

[19] F. Tuinstra and J. L. Koenig, J. Chem. Phys. 53, 1126 (1970).

[20] R. Saito, A. Jorio, A. G. Souza Filho, G. Dresselhaus, M. S. Dresselhaus, and M. A. Pimenta, Phys. Rev. Lett. 87, in press (2001).

[21] C. Thomsen and S. Reich, Phys. Rev. Lett. 85, 5214 (2000).

[22] A. G. Souza Filho, A. Jorio, G. Dresselhaus, M. S. Dresselhaus, A. K. Swan, M. S. Ünlü, B. B. Goldberg, J. H. Hafner, C. M. Lieber, M. A. Pimenta, and R. Saito. In Making Functional Materials with Carbon Nanotubes: MRS Symposium Proceedings, Boston, December 2001, edited by P. Nikolaev, P. Bernier, P. Ajayan, and Y. Iwasa, page Z6.17, Materials Research Society Press, Pittsburgh, PA, 2002.

[23] A. G. Souza Filho, A. Jorio, Ge. G. Samsonidze, G. Dresselhaus, M. S. Dresselhaus, A. K. Swan, B. B. Goldberg, M. S. Ünlü, R. Saito, J. H. Hafner, C. M. Lieber, and M. A. Pimenta, (to be published). 